\title{
Ordering of $\mathrm{TiO}_{2}$-Based Nanostructures on $\mathrm{SrTiO}_{3}(001)$ Surfaces
}

\author{
David S. Deak, Fabien Silly, David T. Newell, and Martin R. Castell* \\ Department of Materials, University of Oxford, Parks Road, Oxford OX1 3PH, United Kingdom
}

Received: February 14, 2006

\begin{abstract}
A class of nanostructured surface phases on $\mathrm{SrTiO}_{3}(001)$ is reported and characterized through atomic-resolution scanning tunneling microscopy and Auger electron spectroscopy. These surface phases are created via argon ion sputtering and UHV annealing and form close-packed domains of highly ordered nanostructures. Depending on the type of nanostructures present, the domain ordering exhibit either $(6 \times 2),(9 \times 2),(12 \times 2),(6 \times 8)$, or $(7 \times 4)$ surface patterning. The nanostructures are composed of $\mathrm{TiO}_{2}$-derived complexes surrounded by a $\mathrm{TiO}_{2}$ surface termination. Such surface ordering phenomena introduce another level of complexity in the chemistry of perovskite oxide surfaces and provide a basis from which potential photocatalytic and molecularordering applications may be developed.
\end{abstract}

\section{Introduction}

Metal oxides play a vital role in a variety of applications ranging from catalysis ${ }^{1}$ to thin-film growth. ${ }^{2,3}$ The chemical properties for a given oxide are strongly linked to its surface structure. Knowing which kinds of surface structures form, and having the ability to control them, are therefore crucial for enhancing current technologies and enabling new ones. Strontium titanate $\left(\mathrm{SrTiO}_{3}\right)$ is an archetypical example of a perovskite structured oxide with elaborate (001) surface structures that have been studied extensively. ${ }^{4-19}$ Interest in $\mathrm{SrTiO}_{3}$ stems from its applications as a substrate for high- $T_{\mathrm{c}}$ superconductors, ${ }^{20}$ in photocatalysis, ${ }^{21}$ in ferroelectrics, ${ }^{22}$ and as a buffer material for micro/nanoelectronic systems. ${ }^{23-25}$ This is because $\mathrm{SrTiO}_{3}$ boasts a number of attractive properties, including a highdielectric constant (high- $\kappa)$ and suitable lattice parameter $(0.3905$ $\mathrm{nm}$ ) for interfacing with other functional materials.

The $\mathrm{SrTiO}_{3}$ crystal consists of $\mathrm{TiO}_{6}$ octahedron units that are connected to each other at the corners forming a cubic lattice. If the corners of the lattice cube intersect at the Ti sites, the $\mathrm{O}$ sites occupy the midpoint of the cube edges. Sr species are found at the center of each cube. When one looks down the [001] direction, the crystal is made of alternating $\mathrm{TiO}_{2}$ and $\mathrm{SrO}$ layers. Although an insulator (3.2 eV band gap), $\mathrm{SrTiO}_{3}$ can become semiconducting upon doping. For example, substitutional $n$ doping of $\mathrm{Nb}^{5+}$ on the Ti sites can make the crystal sufficiently electrically conducting to allow techniques such as scanning tunneling microscopy (STM) or Auger electron spectroscopy (AES) to be performed. Known surfaces of $\mathrm{SrTiO}_{3}(001)$ can be separated into surface reconstructions or non-perovskite surface phases.

Near-stoichiometric reconstructions of $\mathrm{SrTiO}_{3}(001)$ surfaces were first interpreted to be ordered arrays of oxygen vacan$\operatorname{cies}^{12,13}$ formed through a variety of annealing techniques. Now, it is understood that the common $(2 \times 1)$ and $c(4 \times 2)$ surfaces feature a single $\mathrm{TiO}_{2}$ overlayer on top of a $\mathrm{TiO}_{2}$ bulk termination. ${ }^{14-17}$ It is also likely that the $c(4 \times 4)$ and $c(6 \times 2)$ reconstructions have a similar Ti-rich composition. ${ }^{11,18}$ Other near-stoichiometric reconstructions, including the $(\sqrt{ } 5 \times \sqrt{ } 5)$ $R 26.6$ surface and similar "square" configurations, e.g. $(2 \times 2)$

* Corresponding author. E-mail: david.deak@materials.ox.ac.uk. and $(4 \times 4)$, are proposed to be composed of ordered Sr-adatoms on a $\mathrm{TiO}_{2}$ layer. ${ }^{8,19}$

Surface phases are different from standard reconstructed surfaces. They are non-stoichiometric, extend beyond the first couple of monolayers, and are structurally variant to the bulk. Here, we report on a new class of surface phases on $\mathrm{SrTiO}_{3}(001)$. STM reveals that these phases are composed of two-dimensional (2D) domains of highly ordered nanostructures. According to AES, these nanostructures are more Ti-rich than the $(2 \times 1)$ and $c(4 \times 2)$ surfaces $^{14-16}$ and are composed of $\mathrm{Ti}^{4+}$-based species. This study reveals a wealth of unique structures, expanding on the scope of nanostructures reported on previously. ${ }^{26,27}$ Given that titanium dioxide surfaces $^{28}$ are the preferred material for developing photocatalytic applications, the rich collection of $\mathrm{TiO}_{2}$ surface morphologies reported here may provide new means for developing technologies in photocatalysis.

\section{Experimental Setup}

Single crystals of $\mathrm{Nb}$-doped ( $0.5 \%$ weight) $\mathrm{SrTiO}_{3}$ with epipolished surfaces were supplied by PI-KEM UK Ltd., as described previously. ${ }^{26}$ (Samples of 1.0\%-weight La-doping produce similar results.) The samples were introduced into an ultrahigh vacuum (UHV) chamber $\left(10^{-8} \mathrm{~Pa}\right)$ of a STM system (JEOL JSTM4500S) and were degassed through thermal annealing up to $800{ }^{\circ} \mathrm{C}$ for several hours. Annealing was achieved by resistive heating of the semiconducting samples, and temperature measurements were performed through a viewport of the UHV chamber using an optical pyrometer.

The nanostructured surfaces were prepared via argon ion $\left(\mathrm{Ar}^{+}\right)$sputtering followed by a sequence of UHV anneals of the $\mathrm{SrTiO}_{3}(001)$ samples. $\mathrm{Ar}^{+}$sputtering was achieved using a VG Microtech Ion Gun System EX03 which operates at an Ar gas pressure maintained at $\sim 4.5 \times 10^{-4} \mathrm{~Pa}$. Ion beam energies ranged from 0.5 to $3.0 \mathrm{keV}$, and the beam-sample current was kept at around $5 \mu \mathrm{A}$ for 7-15 min. After sputtering, samples were annealed at temperatures ranging from 850 to $1200{ }^{\circ} \mathrm{C}$ for about $30 \mathrm{~min} / \mathrm{anneal}$. By controlling the time, temperature, and number of anneals, it is possible to form particular types of nanostructures and their respective 2D domains on the (001) surface. 
The nanostructured surfaces were imaged in constant current mode using chemically etched tungsten tips in the STM system. AES was performed using a UHV scanning electron microscope (JEOL TM Z9043T) operating at $12 \mathrm{kV}$ accelerating voltage with a $1 \mathrm{nA}$ probe current and a hemispherical electron energy analyzer (SPECS PHOIBOS 150). These instruments are located on part of the STM system. Samples can therefore be transferred into the SEM chamber from the STM chamber without leaving the UHV environment.

\section{Results}

The STM results in this section show six distinctive types of nanostructures that order into close-packed two-dimensional (2D) domains covering all of the $\mathrm{SrTiO}_{3}(001)$ sample surfaces. All domains are oriented in the $\langle 100\rangle$ directions. Once sputtered, samples can subsequently be annealed to produce the desired nanostructured surface. Anneal temperature and time are the critical factors that influence how the nanostructures emerge and evolve. These surfaces and the respective annealing conditions used to obtain them are described as follows.

A sample that is sputtered and then annealed at around 800 ${ }^{\circ} \mathrm{C}$ for 30 min produces a defective and difficult-to-image $(2 \times$ 1) surface. Upon an $850^{\circ} \mathrm{C}$ anneal, surfaces promptly become covered in linear nanostructures, like those shown in Figure 1a. These nanostructures, previously referred to as nanolines, ${ }^{26}$ consist of two rows of protruding atoms or atomic complexes, and will henceforth be referred to as dilines. The ordering found in domains of dilines exhibit a $(6 \times 2)$ surface pattern, as demonstrated by the $2.4 \times 0.8 \mathrm{~nm}^{2}$ rectangle in Figure 1a. When a sample is further annealed at $1000{ }^{\circ} \mathrm{C}$ the surface yields what is shown in Figure 1b. The nanostructures appear to be degenerated dilines, which will be termed meta-dilines. While meta-dilines exhibit the same symmetry and planar dimensions of the dilines (rectangle in Figure 1b), structural features are more subtle and it appears as if the top layer of protruding rows is missing. Figure 1c exemplifies the difference, where the relative corrugation along a diline $(0.2 \mathrm{~nm})$ is more than a metadiline $(<0.1 \mathrm{~nm})$. Meta-dilines maintain a defective periodic pattern of around $0.8 \mathrm{~nm}$ along their length, and a $(6 \times 2)$ pattern of the meta-diline surface is confirmed with low energy electron diffraction (LEED).

Samples that are sputtered and then annealed up to $950{ }^{\circ} \mathrm{C}$ for $30 \mathrm{~min}$ or more host surfaces such as that shown in Figure 2a. These nanostructures are comprised of three rows and are therefore termed trilines. The triline consists of two outer rows, resembling the rows of dilines (Figure 1a), which are separated by a seemingly contiguous "backbone". The rectangle in Figure $2 \mathrm{a}$ indicates the $(9 \times 2)$ symmetry that defines the ordering of the triline domains. Figure $2 b$ is a STM image of a single triline. The protruding atoms/atomic complexes along the outer rows are separated by $0.8 \mathrm{~nm}$ ( 2 unit cells). This is illustrated in the black height profile of Figure 2c. The triline backbone, pointed out by the gray arrow in Figure $2 b$, exhibits periodicity near $0.4 \mathrm{~nm}$ along its length, as indicated by the associated gray profile in Figure 2c. Outer rows have a center-to-center separation of $2.0 \mathrm{~nm}$, or five unit cells, shown in the profile of Figure $2 \mathrm{~d}$. The height of the backbone varies as a function of the imaging bias applied to the sample in the STM. At low sample bias (e.g. $+0.8 \mathrm{~V}$ ), the backbone brightness and height is equal to the height of the outer rows and adjacent diline $(\sim 0.2$ $\mathrm{nm}$ ), as illustrated by the color-enhanced and 3D-rendered STM image of Figure 2e. At high imaging bias (e.g. $2.2 \mathrm{~V}$ ), the backbone increases in height (Figure 2f). This indicates a relative increase in the density of low-energy empty electronic states along the backbone of the triline.
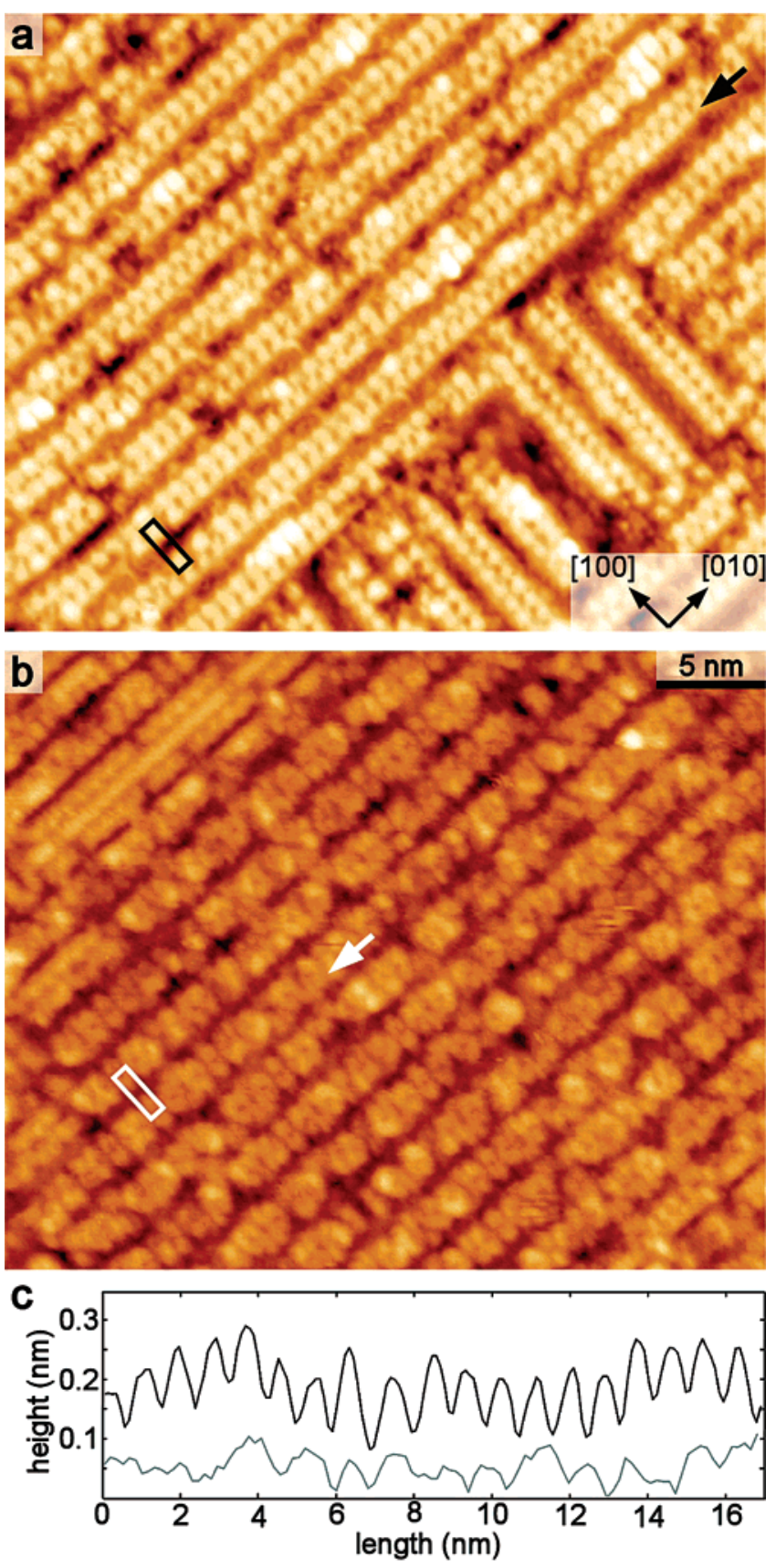

Figure 1. STM data of two different $(6 \times 2)$ surface phases on $\mathrm{SrTiO}_{3}(001)\left(35.0 \times 28.5 \mathrm{~nm}^{2}\right)$ : (a) diline and (b) meta-diline covered surfaces with a $(6 \times 2)$ unit cell drawn (rectangles); (c) relative height profiles along a diline (black) and meta-diline (gray), drawn from where the arrows point in (a) black and (b) white. Image parameters (sample bias, tunneling current): (a) $V_{\mathrm{s}}=+1.4 \mathrm{~V}, I_{\mathrm{t}}=0.10 \mathrm{nA}$; (b) $V_{\mathrm{s}}=+1.1$ $\mathrm{V}, I_{\mathrm{t}}=0.10 \mathrm{nA}$.

Another form of nanostructured surface, shown in Figure 3a, forms domains with a $(12 \times 2)$ surface pattern (see the $4.8 \times$ $0.8 \mathrm{~nm}^{2}$ rectangle in Figure 3a). These nanostructures occupy the width of two dilines and are henceforth termed tetralines. Tetralines are created after sputtering and repeated annealing $(>1 \mathrm{~h})$ at around $1000^{\circ} \mathrm{C}$. The STM image and corresponding profile, shown in Figure 3b,c respectively, illustrate the structural features of a tetraline compared to a triline. With a height over $\sim 0.3 \mathrm{~nm}$, and a width of $3.2 \mathrm{~nm}$ (center-to-center separation of the outer rows), tetralines are noticeably larger than the trilines. While both structures appear to have identical outer rows, the larger tetraline backbone broaden and heighten the structure. The features of the tetraline backbone are difficult to observe 

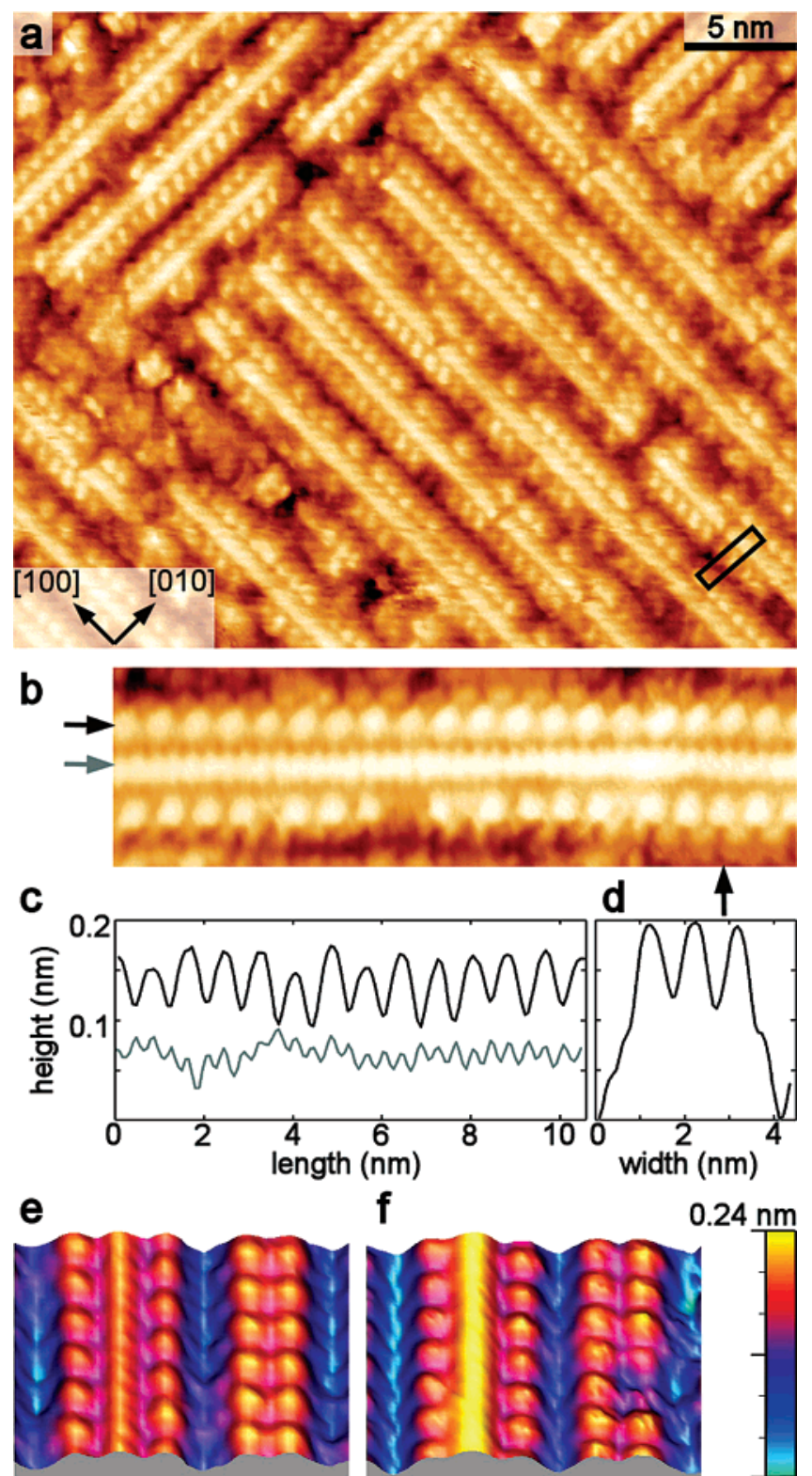

f

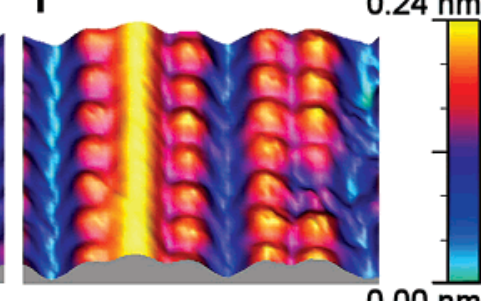

$0.00 \overline{\mathrm{nm}}$

Figure 2. STM data of the $(9 \times 2)$ triline surface of $\mathrm{SrTiO}_{3}(001)$ : (a) image of a triline domain-covered surface with a black $(9 \times 2)$ unit cell (image size: $35.0 \times 28.5 \mathrm{~nm}^{2}, V_{\mathrm{s}}=+1.5 \mathrm{~V}, I_{\mathrm{t}}=0.30 \mathrm{nA}$ ); (b) image of a single triline exhibiting periodic features on the outer rows and the central backbone (image size: $15.5 \times 4.5 \mathrm{~nm}^{2}, V_{\mathrm{s}}=+0.8 \mathrm{~V}$, $I_{\mathrm{t}}=0.30 \mathrm{nA}$ ); (c) profiles taken from where the horizontal arrows point in (b), illustrating the $0.8 \mathrm{~nm}$ periodicity of the outer row (black) and the $0.4 \mathrm{~nm}$ periodicity along the backbone (gray). The relative heights in (c) are offset to visibly separate the profiles; (d) height-width profile of a triline drawn from where the vertical black arrow points in (b), demonstrating a $0.2 \mathrm{~nm}$ height (at $0.8 \mathrm{~V}$ bias) and a $2.0 \mathrm{~nm}$ (5 unit cell) center-to-center separation between outer rows. Similar STM images, (e) and (f), from different regions of the sample $(7.2 \times 5.0$ $\mathrm{nm}^{2}$ ), are rendered in color and 3D. They show an adjacent triline (left) and diline (right), which are imaged at (e) $V_{\mathrm{s}}=+0.8 \mathrm{~V}, I_{\mathrm{t}}=0.30 \mathrm{nA}$ and (f) $V_{\mathrm{s}}=+2.2 \mathrm{~V}, I_{\mathrm{t}}=0.09 \mathrm{nA}$. The triline backbone in (f) is about $0.02 \mathrm{~nm}$ higher than in (e).

along with other features in a single STM image. This is demonstrated in Figure 3d, where particular details of the backbone are resolved. The backbone appears to have densely packed quasi-periodic features and is irregular in morphology.

A sample covered in meta-dilines (Figure 1b) can be repeatedly annealed at temperatures around $950{ }^{\circ} \mathrm{C}$ to produce domains of ordered trenches like those shown in Figure 4. The
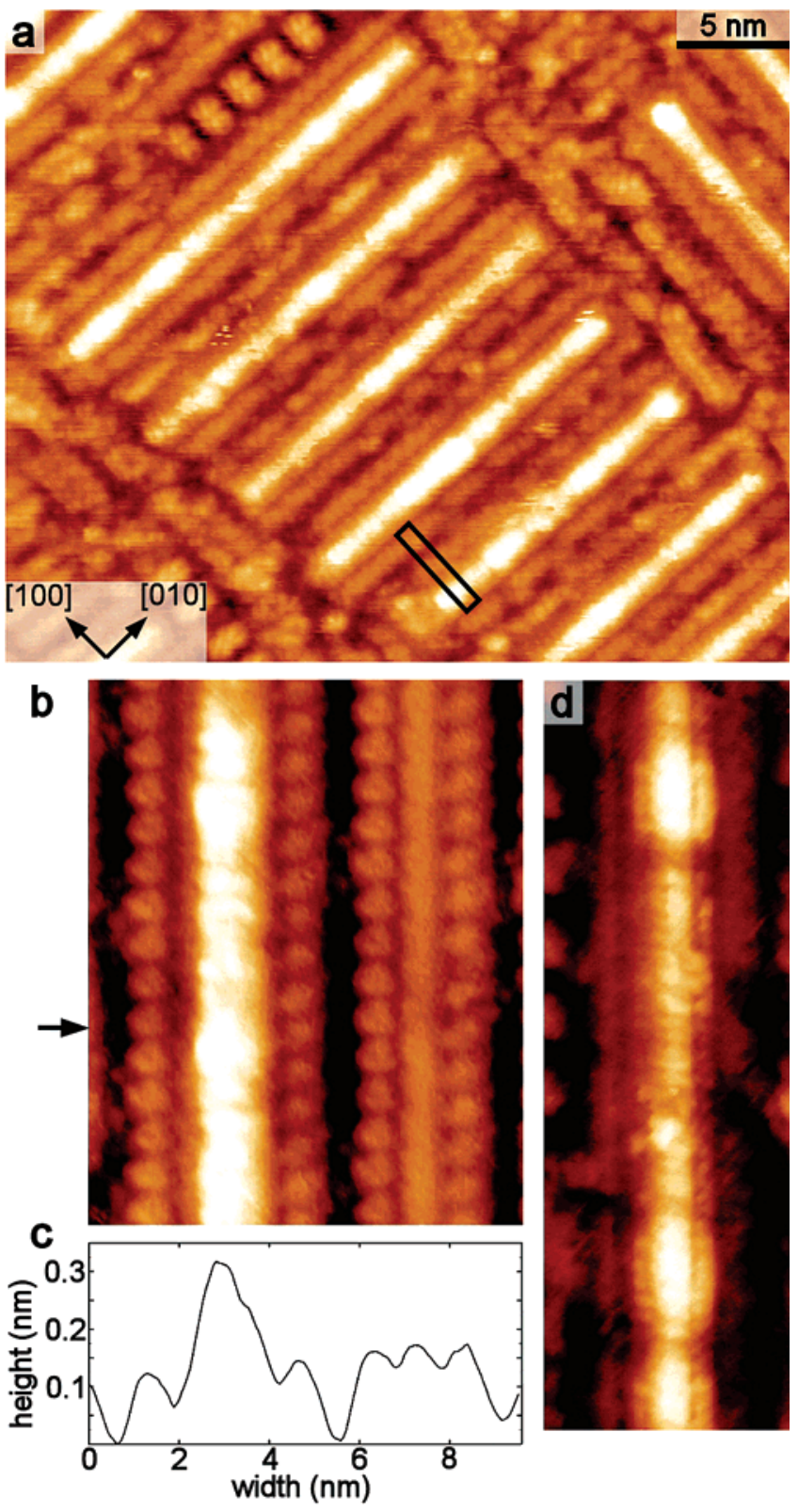

Figure 3. STM data of $(12 \times 2)$ tetraline domains on $\mathrm{SrTiO}_{3}(001)$ : (a) image of a tetraline domain exhibiting a $(12 \times 2)$ surface pattern as indicated by the black unit cell (image size: $35.0 \times 28.5 \mathrm{~nm}^{2}, V_{\mathrm{s}}=$ $+1.7 \mathrm{~V}, I_{\mathrm{t}}=0.30 \mathrm{nA}$; (b) image of an adjacent tetraline (left) and triline (right) $\left(9.6 \times 12.1 \mathrm{~nm}^{2}, V_{\mathrm{s}}=+1.8 \mathrm{~V}, I_{\mathrm{t}}=0.23 \mathrm{nA}\right)$; (c) profile taken from where the arrow points in (b), illustrating the height and width difference between tetralines and trilines; (d) image-resolving features of the tetraline backbone (image size: $6.2 \times 18.7 \mathrm{~nm}^{2}, V_{\mathrm{s}}=$ $+2.1 \mathrm{~V}, I_{\mathrm{t}}=0.30 \mathrm{nA}$ ).

$(6 \times 8)$ pattern produced by these trench domains resemble that of an elongated waffle structure and are often found near stepedges. The trench structures are at least $0.2 \mathrm{~nm}$ deep, and the crosslike features that separate them are of comparable "height" to the meta-dilines. The domains of trench structures are distinctively different from the previously mentioned nanolines (dilines, trilines, and tetralines), in that they are comprised of highly ordered rectangular arrays. The trenches are perfectly aligned in two-dimensions.

Samples that have been sputtered and treated by several 30 min anneals at temperatures ranging from 930 to $970{ }^{\circ} \mathrm{C}$ often produce surfaces with domains of crossdots, as illustrated in Figure 5. Like the waffles (Figure 4), the crossdots (previously termed nanodots ${ }^{26}$ ) are made of cross-shaped units that order 


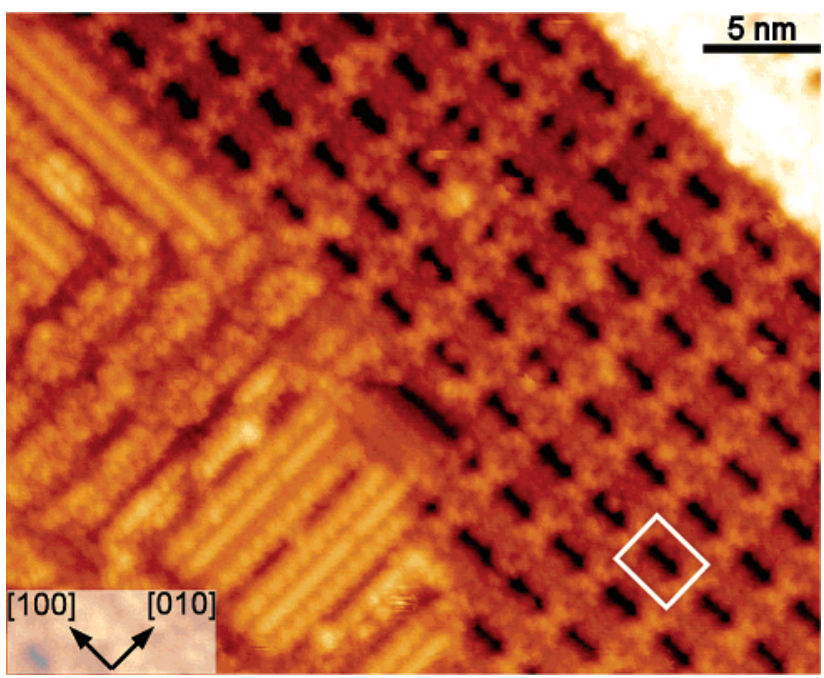

Figure 4. An STM image of trench arrays or "waffles" on $\mathrm{SrTiO}_{3}(001)$ (image size: $35.0 \times 28.5 \mathrm{~nm}^{2}, V_{\mathrm{s}}=+0.8 \mathrm{~V}, I_{\mathrm{t}}=0.30 \mathrm{nA}$ ). The domain exhibits $(6 \times 8)$ periodicity as shown by the white rectangular unit cell $\left(2.4 \times 3.2 \mathrm{~nm}^{2}\right)$.

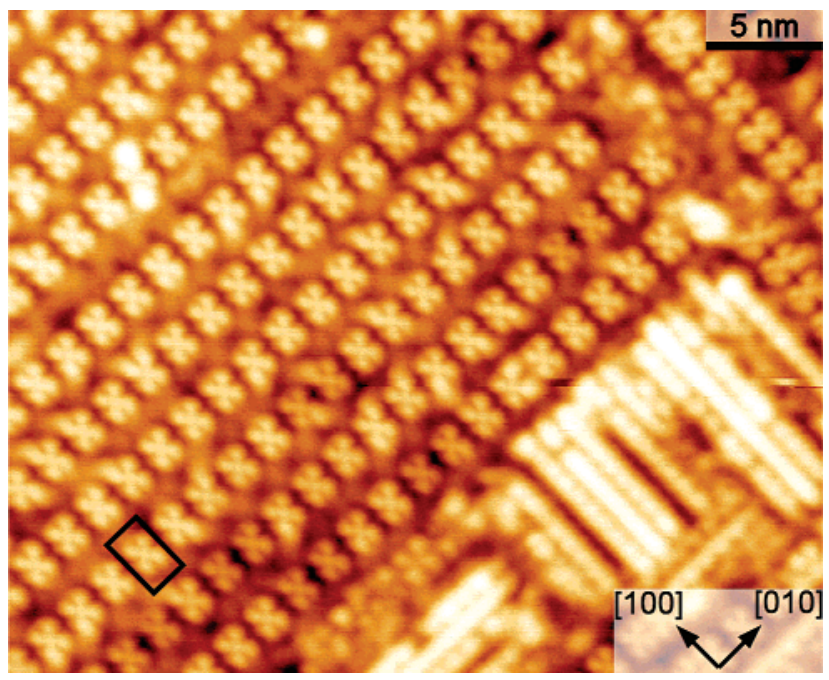

Figure 5. An STM image of a crossdot array on $\mathrm{SrTiO}_{3}(001)$ (image size: $\left.35.0 \times 28.5 \mathrm{~nm}^{2}, V_{\mathrm{s}}=+1.1 \mathrm{~V}, I_{\mathrm{t}}=0.10 \mathrm{nA}\right)$. The black unit cell $\left(2.8 \times 1.6 \mathrm{~nm}^{2}\right)$ shows a $(7 \times 4)$ pattern of the surface domain.

into grids or $2 \mathrm{D}$ arrays, resulting in $(7 \times 4)$ surface symmetry. The crossdots are more close-packed than the trenches in the waffle arrays and exhibit a 4 unit cell periodicity longitudinally (the [010] direction of Figure 5). An interesting feature to note is that while the diline, meta-diline, and waffle domain structure share a 6 unit cell close-packing arrangement between lines/ rows, the crossdot arrays are defined by a wider 7 unit cell periodicity in the [100] direction of Figure 5. Crossdots are 0.2 $\mathrm{nm}$ in height, which is the same as for dilines.

The above six structures are the most common forms of nanostructures found in this class of surface phases on $\mathrm{SrTiO}_{3}(001)$. To determine the chemistry of these nanostructured surfaces, an Auger (AES) spectrum was obtained and compared to AES spectrum of a cleaved $\mathrm{SrTiO}_{3}(001)$ surface, as shown in Figure 6. The UHV SEM was set up to raster scan the electron beam over a $1 \times 1 \mu \mathrm{m}^{2}$ area in the center of the samples. To create a sample that was as close to stoichiometry as possible a crystal was cleaved in air to expose an (001) face, mounted onto a holder, and introduced into the UHV system. It was then degassed at around $100{ }^{\circ} \mathrm{C}$ for $5 \mathrm{~min}$, and a clear $(1 \times 1)$ LEED pattern was observed. The nanostructured surface was produced

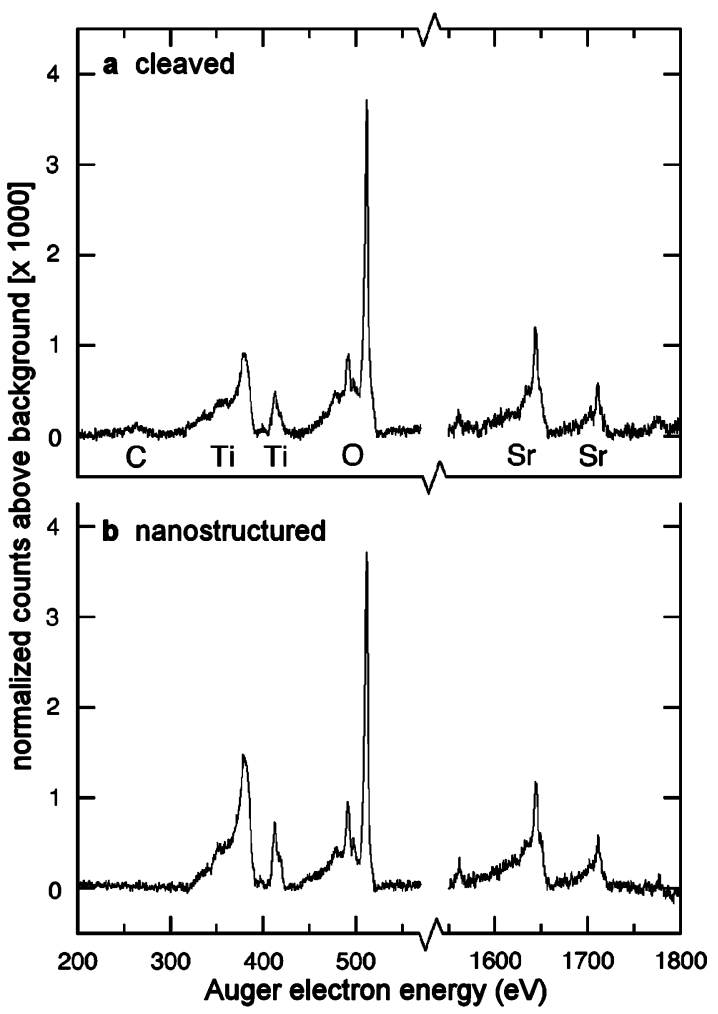

Figure 6. Auger electron spectra of $($ a) a cleaved $(1 \times 1)$ surface and (b) a nanostructure-covered surface of $\mathrm{SrTiO}_{3}(001)$. In (a) the $\mathrm{C}$, Ti, $\mathrm{O}$, and $\mathrm{Sr}$ peaks are indicated. In (b) no $\mathrm{C}$ peak is visible and the $\mathrm{Ti}$ peak is significantly higher than in (a). The profile of the Ti peak at $418 \mathrm{eV}$ is a signature for $\mathrm{Ti}^{4+}$. The spectra were normalized to have a common $\mathrm{O}$ peak height. The featureless region between 570 and 1550 $\mathrm{eV}$ has been omitted.

by sputtering and annealing the sample at $970{ }^{\circ} \mathrm{C}$ for about 1 $\mathrm{h}$, and according to STM, the surface was fully covered in dilines, trilines, and crossdot arrays. Auger data is often presented as the differential of the signal to enhance the peaks. However, this process is not necessary in our system because the signal-to-noise ratio is sufficiently high to show the peaks directly.

AES spectra from a cleaved $(1 \times 1)$ and nanostructured covered $\mathrm{SrTiO}_{3}(001)$ sample are shown in Figure 6a,b, respectively. To facilitate comparisons of spectra a background subtraction was performed and the spectra were normalized so that they have the same $\mathrm{O}$ peak height at $510 \mathrm{eV}$. The most striking difference between the spectra is the enhancement of the Ti peaks at 387 and $418 \mathrm{eV}$ for the nanostructured surface (this peak enhancement is not as obvious for $(2 \times 1)$ or $c(4 \times$ 2) surfaces). Also, the profile of the $418 \mathrm{eV} \mathrm{Ti}$ peak is a signature for a surface that is composed of $\mathrm{Ti}^{4+}$ species, as apposed to $\mathrm{Ti}^{2+}$ species. It is worth noting the small $\mathrm{C}$ peak at $272 \mathrm{eV}$ from the cleaved sample. This is due to contamination arising from the brief exposure to hydrocarbons in the ambient environment following sample cleavage. The two main $\mathrm{Sr}$ peaks are at 1649 and $1717 \mathrm{eV}$.

\section{Discussion}

To prepare the nanostructured surfaces it is necessary to sputter the sample before annealing. The $\mathrm{Ar}^{+}$sputtering process creates the defects and/or surface morphology that allow the constituents of the nanostructures to diffuse to and assemble on the surface during the annealing process. Repeated annealing after sputtering results in surface transformations from one dominating nanostructure to another. The first structure to 
emerge, at the lowest annealing temperatures, is the diline. Higher temperatures produce trilines, which may require higher energies to form the larger and more complex structure. Tetralines are even larger structures, and require yet even higher temperatures and/or annealing time to form. It was previously indicated that dilines evolve into crossdots ${ }^{26}$ (formerly nanolines and nanodots). For this transformation to occur within domains, where a $(6 \times 2)$ pattern changes to a $(7 \times 4)$ pattern, longitudinal conjugations and transverse separations must be part of the mechanism. Conversely, if meta-diline domains or waffle structures are to evolve from diline domains, only longitudinal transformations are likely to feature.

The STM data demonstrate that all six structures share common attributes, as well as differentiating features. Dilines, trilines, and tetralines (collectively called nanolines) are framed with what seems to be a pair of single rows of protruding atomic complexes. These single rows are presumably composed of $\mathrm{TiO}_{2}$-based species. Within each nanoline, row pairs are observed in zigzag or square configurations (previously discussed for dilines ${ }^{26}$ ). The height of these rows do not change significantly as a function of imaging bias or tunneling current in the STM. This indicates that the structural features shown in STM images are mostly due to topographic effects. On the other hand, the height of the backbone of the triline structures does vary with the imaging bias. This signifies a dominating electronic effect which convolutes the topographic features of the backbone. Tetralines host a backbone that is invariant with height under different imaging conditions but features irregular structure and is densely packed.

Structural features of the meta-dilines suggest that they are dilines which are missing the top layer of protruding atomic complexes: what is left is the underlying residual structure that maintains the $(6 \times 2)$ surface pattern. The emergence of waffle structures may be the result of further degeneration of metadiline domains, where the 6 unit cell periodicity between closepacked trench lines is maintained. Lengthwise, however, crosslike structures separate the individual trenches creating the 8 unit cell translational symmetry. Crossdot lines and arrays appear to be a transformed-not degenerated-product of dilines. The crosses protrude out of the surface with the same $0.2 \mathrm{~nm}$ height as the dilines. It is possible that these crossdots are made of the same protruding atomic complexes found in nanolines but with a different structure that exhibits itself as the crosslike protrusions. A remarkable feature of the crossdots and the trenches is how they align in two dimensions to create perfectly ordered rectangular arrays.

All the nanostructured surfaces form under similar preparation conditions and emerge on a precursor surface with a $(2 \times 1)$ reconstruction or in the case of previous study ${ }^{26}$ a $c(4 \times 2)$ reconstruction. Both precursor surfaces are constructed from a $\mathrm{TiO}_{2}$-rich overlayer on a $\mathrm{TiO}_{2}$ termination. AES spectra for the nanostructured surface suggest an overwhelmingly Ti-rich construction, made of $\mathrm{Ti}^{4+}$ species, and hence is $\mathrm{TiO}_{2}$-based. If the $\mathrm{Ti} / \mathrm{Sr}$ peak ratio from the cleaved sample (Figure 6a) is assumed to represent $\mathrm{SrTiO}_{3}$ stoichiometry, then the nanostructure-covered sample (Figure $6 \mathrm{~b}$ ) shows a molar Ti/Sr ratio of 1.68 , i.e., a substantial increase in the amount of $\mathrm{Ti}$ in the surface region. Therefore, these nanostructured surfaces can be said to be a class of titanium oxide surface phases on $\mathrm{SrTiO}_{3}(001)$. Related surface structures, such as those found on $\mathrm{TiO}_{2}$ crystals, ${ }^{29}$ may provide a basis from which theoretical models of these nanostructures could be developed.

Surfaces that are annealed for long periods of time $(>1 \mathrm{~h})$ and/or high temperatures $\left(>1000{ }^{\circ} \mathrm{C}\right)$ are mostly covered in degenerate forms of nanostructures, such as the meta-dilines and waffle structures. Such surfaces often host tetralines. This "degeneration" occurs in conjunction with the observance of anatase islands. ${ }^{30}$ Small anatase islands have been found near and on tetralines. Consequently, it may be the case that tetralines are a precursor structure or nucleation site for anatase nanoisland growth. In addition, the protrusions found on dilines, which are missing on meta-dilines, could be the $\mathrm{TiO}_{2}$ building blocks required for the creation of anatase nanoislands.

\section{Conclusion}

In summary, samples of $\mathrm{SrTiO}_{3}(001)$ that are $\mathrm{Ar}^{+}$sputtered and subsequently annealed at temperatures between 850 and $1200{ }^{\circ} \mathrm{C}$ produce nanostructured surface phases that are composed of $\mathrm{TiO}_{2}$-related species. The surface nanostructures, which exhibit highly periodic features, are uniform and order into close-packed domains which are oriented in the $\langle 100\rangle$ crystallographic directions. The domains cover the entire surface of the sample crystal. Six different kinds of nanostructures are observed: dilines, trilines, tetralines, crossdots, meta-dilines, and trenches. Dilines, trilines, and tetralines form nanoline domains, whereas the crossdots and trenches order into rectangular arrays with features aligning in 2D. Evidence suggests that the nanostructures degenerate after extended annealing in conjunction with the formation of anatase islands, which further supports the AES analysis that the nanostructures are composed of $\mathrm{TiO}_{2}$ units.

The high degree of ordering and the suitable length scales on which these nanostructures form are key attributes that can enable these surface phases to act as a template for ordering molecules in new and unique ways. $\mathrm{SrTiO}_{3}$ and $\mathrm{TiO}_{2}$ are also known to have attractive photocatalytic properties. It is therefore proposed that these $\mathrm{TiO}_{2}$-based surface phases on $\mathrm{SrTiO}_{3}$ may also prove useful in developing photocatalytic technologies.

Acknowledgment. The authors thank the Royal Society, the EPSRC, JEOL UK, and DSTL for funding and Chris Spencer (JEOL UK) for precious technical support.

\section{References and Notes}

(1) Keane, M. A. J. Mater. Sci. 2003, 38, 4661.

(2) Gupta, A. Curr. Opin. Solid State Mater. Sci. 1997, 2, 23.

(3) Chambers, S. A. Surf. Sci. Rep. 2000, 39, 105.

(4) Matsumoto, T.; Tanaka, H.; Kawai, T.; Kawai, S. Surf. Sci. 1992, 278, L153-L158.

(5) Liang, Y.; Bonnell, D. J. Am. Chem. Soc. 1995, 78, 2633-2640.

(6) Jiang, Q.; Zegenhagen, J. Surf. Sci. 1996, 367, L42-L46.

(7) Charlton, G.; Brennan, S.; Muryn, C. A.; McGrath, R.; Norman, D.; Turner, T.; Thornton, G. Surf. Sci. 2000, 457, L376-L380.

(8) Kubo, T.; Nozoye, H. Phys. Rev. Lett. 2001, 86, 1801.

(9) Reagor, D. W.; Butko, V. Y. Nat. Mater. 2005, 4, 593-596.

(10) Kan, D. S.; Terashima, T.; Kanda, R.; Masuno, A.; Tanaka, K.; Chu, S. C.; Kan, H.; Ishizumi, A.; Kanemitsu, Y.; Shimakawa, Y.; Takano, M. Nat. Mater. 2005, 4, 816-819.

(11) Erdman, N.; Marks, L. D. Surf. Sci. 2003, 526, 107.

(12) Cord, B.; Courths, R. Surf. Sci. 1985, 162, 34.

(13) Tanaka, H.; Matsumoto, T.; Kawai, T.; Kawai, S. Jpn. J. Appl. Phys. 1993, 36, 1405.

(14) Erdman, N.; Poeppelmeier, K. R.; Asta, M.; Warschkow, O.; Ellis, D. E.; Marks, L. D. Nature 2002, 419, 55.

(15) Erdman, N.; Warschkow, O.; Asta, M.; Poeppelmeier, K. R.; Ellis, D. E.; Marks, L. D. J. Am. Chem. Soc. 2003, 124, 10050.

(16) Warschkow, O.; Asta, M.; Erdman, N.; Poeppelmeier, K. R.; Ellis, D. E.; Marks, L. D. Surf. Sci. 2004, 573, 446.

(17) Johnston, K.; Castell, M. R.; Paxton, A. T.; Finnis, M. W. Phys. Rev. B 2004, 70, 085415 .

(18) Castell, M. R. Surf. Sci. 2002, 505, 1.

(19) Kubo, T.; Nozoye, H. Surf. Sci. 2003, 542, 177.

(20) Aruta, C. Phys. Status Solidi A 2001, 183, 353.

(21) Yin, J.; Ye, J.; Zou, Z. Appl. Phys. Lett. 2004, 85, 689

(22) Ahn, C. H.; Rabe, K. M.; Triscone, J. M. Science 2004, 303, 488. 
(23) Först, C. J.; Ashman, C. R.; Schwarz, K.; Blöchl, P. E. Nature 2004, 427, 53.

(24) Liang, Y.; Kulik, J.; Eschrich, T. C.; Droopad, R.; Yu, Z.; Maniar, P. Appl. Phys. Lett. 2004, 85, 1217.

(25) Kim, B. M.; Brintlinger, T.; Cobas, E.; Fuhrer, M. S.; Zheng, H. M.; Yu, Z.; Droopad, R.; Ramdani, J.; Eisenbeiser, K. Appl. Phys. Lett. 2004, 84, 1946.
(26) Castell, M. R. Surf. Sci. 2002, 516, 33.

(27) Silly, F.; Castell, M. R. J. Phys. Chem. B 2005, 109, 12316.

(28) Diebold, U. Surf. Sci. Rep. 2003, 48, 53.

(29) Bennett, R. A.; Stone, P.; Price, N. J.; Bowker, M. Phys. Rev. Lett. 1999, 82,3831 .

(30) Silly, F.; Castell, M. R. Appl. Phys. Lett. 2004, 85, 3223. 